dos mecanismos moleculares com importância na instalação e progressão da doença.

Materiais e métodos: Efetuou-se uma revisão bibliográfica dos estudos de proteoma existentes sobre peri-implantite, utilizando as bases de dados Medline e as palavras-chave 'peri-implantitis", "biomarkers", "proteome", "bone diseases" e "dental implants" combinadas com o operador booleano "AND". A informação recolhida foi anotada de forma manual na base de dados SalivaTecDB e, subsequentemente, foi realizada a caracterização funcional do OralOma da peri-implantite à luz do conhecimento existente relativo a indivíduos saudáveis e com periodontite, com recurso a estratégias bioinformáticas.

Resultados: Este trabalho permitiu incrementar de 38 para 96 o n..$^{\circ}$ de proteínas na base de dados SalivaTecDB para esta patologia. A maioria das proteínas catalogadas apresentavam dados de quantificação, contudo, não existe homogeneidade quanto às unidades utilizadas e aos métodos de recolha e análise. As análises funcionais permitiram elucidar alguns dos mecanismos moleculares comuns entre a peri-implantite e a periodontite. $\mathrm{O}$ aumento das concentrações de IL-1b, MPO e TNF-a e a diminuição de IL-10 poderão refletir a fase inicial da patologia peri-implantar em que é estimulado o recrutamento de células. $O$ aumento da MMP-8 reflete o início da fase de destruição de tecidos peri-implantares e finalmente, o aumento do RANK, RANKL e a diminuição da OPG são indicadores da osteoclastogénese, processo essencial para a instalação da doença.

Conclusões: Este estudo mostra que apesar da utilização de OPG recombinante poder ser uma opção para travar a reabsorção óssea, como tem sido sugerido noutros trabalhos, esta seria uma solução a curto prazo mas que não reverteria a doença, uma vez que estaríamos a atuar no final do processo. Neste sentido, sugere-se que a intervenção terapêutica seja feita no início do mesmo, antes da produção de mediadores inflamatórios, pelo que se propõe que a modelação das células imunes seja estudada em trabalhos futuros de modo a identificar os melhores alvos terapêuticos.

http://doi.org/10.24873/j.rpemd.2017.12.149

\#127 Alterações musculo-esqueléticas orofaciais em doentes com artrite reumatóide

Helena Campos Silva*, Francisco Maligno, Catarina Aguiar Branco, João Carlos Pinho

Faculdade de Medicina Dentária da Universidade do Porto

Objetivos: Identificação das alterações músculo-esqueléticas orofaciais mais frequentes em doentes com Artrite Reumatóide

Materiais e métodos: No presente estudo foram incluídos 38 doentes $(n=38)$ com Artrite Reumatóide e utentes do Centro Hospitalar de Entre o Douro e Vouga. Os voluntários foram submetidos a um questionário e exame clínico, o Research Diagnostic Criteria for Temporomandibular Disorders. O exame clínico foi efetuado sempre pelo mesmo examinador e numa sala com temperatura controlada. Foi também efetuado um exame ecodoppler da articulação temporomandibular. Para cada doente foram captadas 4 imagens ultrassonográficas, com boca aberta e fechada, à esquerda e à direita. Os parâmetros analisados foram cinco: deformidade e contorno da superfície do côndilo, presença de erosões, aumento da vascularização sinovial e dimensão do espaço articular.

Resultados: Com esta investigação, foi possível verificar que $63 \%$ dos doentes apresentam dor facial. Em $42 \%$ dos casos a dor foi bilateral, surgindo apenas à direita em 10\% dos doentes e à esquerda em 11\%. A origem da dor facial foi maioritariamente relacionada com a associação entre os componentes muscular e articular, quer à esquerda (40\%), quer à direita (50\%). Isoladamente, a mialgia foi mais frequente do que a artralgia. Os músculos mais afetados foram o corpo do masséter (extra oral) e a área onde se insere o ptérigoideu lateral superior (intraoral). O polo lateral da articulação temporomandibular foi o mais doloroso. Foi encontrada uma relação estatisticamente significativa ( $\mathrm{p}>0.001)$ entre a dor à palpação e a limitação da abertura da boca. Alterações ósseas da superfície condilar surgiram em $100 \%$ dos doentes com estadio grave de artrite reumatóide.

Conclusões: Neste estudo, os doentes com artrite reumatóide apresentaram, frequentemente, alterações músculo-esqueléticas. Os componentes muscular e articular estiveram praticamente envolvidos de forma simultânea. Foram frequentemente encontradas alterações da superfície condilar. É de grande interesse para o médico dentista que sejam reconhecidos os locais dolorosos e as alterações estruturais mais frequentes neste tipo de doentes, de forma a que seja possível uma abordagem mais efetiva e benéfica, contribuindo para que sejam aprimoradas e individualizadas técnicas que permitam minimizar os efeitos orofaciais da artrite reumatóide.

http://doi.org/10.24873/j.rpemd.2017.12.150

\#128 Dermatophagoides pteronyssinus induz células CD4 RORgt FoxP3 em crianças asmáticas

Gabriela Alberis Marques*, Patricia Dias de Araujo

Faculdade Especializada na Área da Saúde do Rio Grande do Sul, Instituto de Pesquisas Biomédicas, Pontifícia Universidade Católica do Rio Grande do Sul (PUCRS)

Objetivos: Avaliar as células que expressam os fatores transcricionais do mestre regulador (MRTF) no sangue periférico de crianças asmáticas atópicas para D.pteronyssinus com ou sem estimulação in vitro com Der p 1.

Materiais e métodos: 129 crianças asmáticas de 8 a 14 anos de idade foram matriculadas no estudo. O estado atópico foi definido pela medição de IgE específica no soro. As PBMCs foram isoladas e estimuladas com Der $\mathrm{p} 1$, anticorpos anti-CD3 / anti-CD28 ou não foram estimuladas por 24 horas. A expressão de T-bet, GATA-3, RORgt e FoxP3 foi analisada por citometria de fluxo.

Resultados: Descobrimos que 85,4\% das crianças asmáticas apresentaram IgE específica para D.pteronyssinus. As crianças atópicas para D.pteronyssus apresentaram alta freqüência de células CD4 RORgt GATA-3 FoxP3 no sangue peri- 
férico. A frequência das células CD4 RORgt, CD4 CD25 FoxP3 e CD4 RORgt FoxP3 aumentou quando PBMCs dessas crianças foram estimuladas com Der p 1 em comparação com a estimulação com anti-CD3 / anti-CD28 ou controle.

Conclusões: Nossos resultados sugeriram que o alérgeno tem um papel no desenvolvimento da célula que expressa mais de Um MRTF.

http://doi.org/10.24873/j.rpemd.2017.12.151

\section{\#129 Otimização do diagnóstico periodontal num registo clínico electrónico}

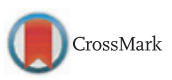

Luís Clemente*, Manuela Romano, Tiago Marques, André Correia

Unidade Local de Saúde de Castelo Branco - Hospital

Amato-Lusitano, Instituto Ciências da Saúde, Viseu, Universidade Católica Portuguesa

Objetivos: Otimização de um registo clínico eletrónico em ambiente universitário com inclusão de um módulo de classificação de diagnóstico periodontal; realização de uma casuística de patologias periodontais no distrito de Viseu através do registo clínico electrónico.

Materiais e métodos: Numa primeira fase, formulou-se um modelo conceptual para desenvolvimento de um registo clínico electrónico de patologias periodontais, baseado na classificação de diagnóstico periodontal de Armitage GC (1999). Esse modelo foi discutido, desenvolvido e implementado com a equipa técnica do programa Newsoft ${ }^{\circledR}$, utilizado na Clínica Dentária Universitária da Universidade Católica Portuguesa, em Viseu. Após implementação dessa ferramenta informática, desenvolveu-se um estudo prospetivo, com uma recolha de dados clínicos periodontais realizada entre o período de 22 de Fevereiro e 10 de Maio de 2017, que incluiu todos os doentes residentes no distrito de Viseu que recorreram à clinica para tratamentos periodontais. Foram analisadas as variáveis: código postal, idade, género, hábitos tabágicos, patologia cardiovascular, hipertensão arterial, diabetes e sua tipologia e o diagnóstico periodontal.

Resultados: O módulo informático de registo do diagnóstico periodontal foi implementado no sistema de registo clínico eletrónico da clínica universitária. De 158 pacientes incluídos no estudo, apenas 134 apresentavam registo das variáveis em análsie. A média de idades registada foi de 52 anos. Os diagnósticos mais frequentes foram as periodontites (crónica) seguidas das doenças gengivais (gengivite induzida por placa). As periodontites foram registadas em idades mais avançadas que as doenças gengivais $(\mathrm{p}<0,01)$. A presença de patologia cardiovascular $(p=0,04)$ assim como a HTA $(p=0,03)$ mostrou uma maior relação com a periodontite em comparação com as doenças gengivais. Não se encontrou diferença significativa quanto aos hábitos tabágicos e a presença ou tipo de diabetes. Quanto à gravidade da periodontite crónica verificou-se uma relação estatisticamente significativa com o aumento da idade $(\mathrm{p}<0,01)$.

Conclusões: A implementação de um módulo de diagnóstico periodontal no registo clínico electrónico permitiu optimizar o seu funcionamento, pois este parâmetro é fundamental em qualquer consulta de Medicina Dentária. No estudo dos efeitos desta implementação foi possível efetuar um levantamento epidemiológico da patologia periodontal no distrito de Viseu. http://doi.org/10.24873/j.rpemd.2017.12.152

\section{\#130 Pesquisa de informação de saúde oral pelos} pacientes de uma clínica dentária universitária

A.R. Oliveira*, B. Oliveira, H. Costa, A. Correia

Universidade Católica Portuguesa, Instituto de Ciências da Saúde -Viseu, Portugal

Objetivos: Determinar o uso da Internet por parte dos pacientes de uma clínica dentária universitária para obterem informações sobre a sua saúde oral e necessidades de tratamento.

Materiais e métodos: Foi aplicado um questionário (adaptado de Riordáin, 2009) aos pacientes da Clínica Dentária Universitária da Universidade Católica Portuguesa, em Viseu, entre 17 de Março e 7 de Abril de 2017. Os dados recolhidos foram sujeitos a análise utilizando o software Excel ${ }^{\circledR}$ Micosoft.

Resultados: A amostra obtida foi constituída por 100 indivíduos. Verificou-se que $50 \%$ dos pacientes tem acesso à Internet diariamente, mas apenas $43 \%$ dos pacientes procurou ou teve um familiar ou amigo que procurasse sobre a sua condição oral. O Google foi o motor de busca mais utilizado $(37,1 \%)$, seguido da pesquisa quer no Google quer no Youtube $(9,7 \%), 8,1 \%$ dos inquiridos pesquisaram no Youtube e, apenas, 3,2\% dos pacientes obteve informação no Google Académico. A qualidade de informação obtida foi moderada para 40,3\% dos pacientes, sendo que $11,3 \%$ considerou a qualidade baixa e, somente, $6,5 \%$ considerou elevada. De notar que, $41,9 \%$ dos pacientes que têm acesso no seu dia a dia à Internet não faz qualquer pesquisa acerca da sua condição de saúde oral. Relativamente à hipótese de consultar um médico dentista pela Internet 34\% considera essa hipótese, enquanto que apenas $23 \%$ considera a possibilidade de realizar um tratamento no estrangeiro.

Conclusões: A Internet parece representar uma ferramenta de obtenção de informação relevante. A relação médico dentista/paciente modificou-se desde o aparecimento da Internet. A informação disponível nos nossos dias é muito variada e os pacientes não têm, muitas vezes, a capacidade de selecionar a informação correta. Assim, o médico dentista deve estar sensibilizado para esta questão e ser capaz de corrigir a desinformação do paciente.

http://doi.org/10.24873/j.rpemd.2017.12.153

\section{\#132 Influência do protocolo de queima} na transmitância de restaurações de cerâmica

João Carlos Roque*, João Paulo Martins, Luís Santos, Jaime Portugal

Instituto Superior Técnico da Universidade de Lisboa, Faculdade de Medicina Dentária da Universidade de Lisboa

Objetivos: Investigar in vitro a influência da alteração do protocolo de queima na transmissão direta de luz (T\%) de restaurações de cerâmica fabricadas com diversas infraestruturas de materiais cerâmicos de alta resistência. 